\title{
Estimation of Battery Health based on Improved Unscented Kalman Filtering Algorithm
}

\author{
Haiying Wang ${ }^{*}$, Yu Wang, Zhilong Yu, and Ran Li \\ School of Automation, Harbin University of Science and Technology, Harbin, 150080, China
}

\begin{abstract}
In connection with the life aging problem of valve-regulated lead-acid batteries (VRLA), to ensure that batteries have a good performance and long life, we have considered VRLA as the backup power that works in a complex environment for a long time. The noise signal is analyzed, a VRLA health estimation model of the double adaptive Kalman algorithm is built, and a method of estimating battery health based on the improved unscented Kalman filter is put forward by using the battery Thevenin equivalent circuit model. The test results show that the average relative error of the VRLA health state estimated by the improved UKF algorithm is $3.1 \%$ and it can estimate the VRLA health state effectively.
\end{abstract}

Keywords: VRLA; Kalman algorithm; state of health; internal resistance

(Submitted on December 18, 2018; Revised on January 16, 2019; Accepted on February 12, 2019)

(C) 2019 Totem Publisher, Inc. All rights reserved.

\section{Introduction}

Batteries will become degraded due to long-time loss and aging accumulation. Thus, the effective real-time monitoring of the VRLA state of health, SOH, is the most concerned problem of users. If batteries degrade and workers are not reminded to replace them in time, the UPS system cannot be ensured to work normally. This may also cause significant losses to companies [1-2]. It is necessary to predict the battery SOH online accurately to allow the battery SOH to be shown directly. The estimation of battery SOH real-time monitoring systems is also weak. This may not only make it impossible to estimate the capacity of batteries but also affect the reliability and safety of batteries directly if the battery SOH is estimated inaccurately. In the end, the real-time monitoring system of batteries cannot evaluate the battery performance effectively, and it may also have a large impact on the battery performance and life [3-4].

At present, many scholars study the SOC and SOH of batteries [5-9]. The important index of battery state of health can be standardized by Ohm resistance, which is one of the battery performance parameters. Under most conditions, the VRLA whose resistance is smaller between two batteries has a stronger discharge capacity. We can reach the conclusion from the cycle charge and discharge life experiment that Ohm resistance affects the SOH of VRLA [10]. Thus, the state of health, $\mathrm{SOH}$, is mainly predicted by resistance. At the same time, the resistance is affected by voltage and SOC value, so therefore they affect SOH indirectly [11].

To address this problem, this paper puts forward a method of estimating battery state of health based on improved unscented Kalman filtering. First, analyze the characterization and influence factors of SOH through related tests and determine that the battery resistance will increase at the float state and the accuracy of battery state of health estimation may be affected by SOC. Then, improve the unscented Kalman filtering algorithm from two aspects of state noise and observe interference, and then get the double adaptive unscented Kalman filtering algorithm of this paper. Theoretical simulation verifies that the battery state of health prediction of this algorithm is very accurate.

* Corresponding author.

E-mail address: wanghy@hrbust.edu.cn 


\section{SOH Estimation of VRLA}

The Thevenin equivalent model is one of the battery models. There are many advantages to choose it as the battery model to establish the state equation. For example, it can reflect the static and dynamic characteristics of batteries and the amount of computing is relatively small. This battery equivalent model is a first order model, and the circuit construction form is shown in Figure 1. In the circuit of Figure $1, U_{P}$ is the battery polarization voltage, $U_{O C}$ is the battery open circuit voltage, $R_{0}$ is the battery Ohm resistance, $C_{P}$ is the battery polarization capacitance, and $R_{P}$ is the polarization resistance, which is in parallel with polarization capacitance $C_{P}$.

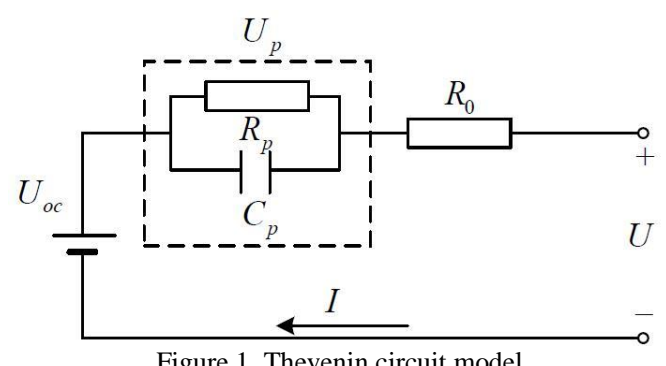

Figure 1. Thevenin circuit model

The variance of the equivalent circuit model is shown as follows:

$$
\begin{gathered}
U(t)=U_{O C}(S O C, T)-U_{P}(t)-i(t) \times R_{0}(S O C, T, I) \\
i(t)=\frac{U_{P}(t)}{R_{P}}+C_{P} \frac{\mathrm{d}\left(U_{P}(t)\right)}{\mathrm{d} t}
\end{gathered}
$$

\section{Method of Estimating SOH based on Improved Unscented Kalman Filtering}

Under practical sampling filtering strategy, unscented Kalman filtering, UKF, is widely used. However, we have to solve the following disadvantages for this algorithm [12-13]:

1) Adaptive ability. Algorithm performance is affected by state fluctuation, which is caused by noise under the nonstationary environment and convergence speed. The change of UKF algorithm performance needs to be counted according to the change of the environment. The weighted combination of measurement estimates and forecast estimates can obtain the best state of the UKF algorithm in dynamic systems. However, the mathematical model is obtained approximately by using real physical models of dynamic systems so that we cannot avoid errors. In this way, we cannot count the noise characteristics, so the Kalman filtering algorithm tends to be divergent in the application process. By analyzing the recursive process of the UKF algorithm, we can determine that the estimation in the filtering and the real error variance is caused by model error. Model error in UKF is not affected by gain and error variance. Finally, the gain of the measured data becomes smaller, the filter diverges, and the data tends to saturation [14-15].

2) Robustness. Filter divergence is also caused by internal and external noise interference, and the filter with the ability to handle interference is weak and may weaken accuracy and stability. Theoretically, the UKF filter should have better accuracy and stability in the condition of robustness [16-17]. The two characteristics cannot cause large errors due to small interference.

Thus, this paper improves the unscented Kalman filtering algorithm in two aspects of observation interference and state noise [18]. Using the introduction of the adaptive matrix and factor, the rules of noise appearance are monitored respectively. Smooth the filter and perform Gaussian processing to reduce the interference of noise to filter in the end.

\subsection{State Noise Improvement}

To make the derivation of additive noise more understandable, we assume that the observation equation is linear [19]. Then $\hat{P}_{k}^{-}$is innovation covariance, which is the sequence of the prediction residual in the UKF algorithm. 


$$
\hat{P}_{k}^{-}=\frac{1}{m} \sum_{j=1}^{m}\left(\hat{x}_{k}-\hat{x}_{k}^{-}\right)\left(\hat{x}_{k}-\hat{x}_{k}^{-}\right)^{\mathrm{T}}
$$

The prediction variance $P_{k}$ obtained from Equation (3) is the counting innovation covariance $P_{k}^{\wedge}{ }^{-}$. Considering the importance of variance prediction, this section checks if we need the zoom state noise variance. We need to compare the changes of state noise variance obtained from $\hat{P}_{k}^{-}$and ${P_{k}^{\wedge}}^{-}$.

Innovation is produced from the observation equation.

$$
d_{k}=\left(H_{k} x_{k}+v_{k}\right)-H_{k} \hat{x^{-}}=H_{k}\left(x_{k}-\hat{x_{-}^{-}}\right)+v_{k}
$$

Assume the situation is filtering optimal, the variable of the Gaussian white noise sequence, whose mean is 0 , is $d_{k}$. Extract the root on each side of the equal sign of the above formula and take the variance [20].

$$
\frac{1}{m} \sum_{j=1}^{m} d_{k-j} d_{k-j}^{\mathrm{T}}=H_{k} \hat{P}_{k}^{-} H_{k}^{\mathrm{T}}+R_{k}
$$

Though $\hat{P}_{k}^{-}$cannot be obtained from Equation (3), $\hat{P}_{k}^{-}$is regarded as the criteria for judging the estimation of state noise variance. Add scale factor $\beta_{k}$.

$$
\beta_{k}=\frac{\operatorname{tr}\left(H_{k} \hat{P}_{k}^{-} H_{k}^{\mathrm{T}}\right)}{\operatorname{tr}\left(H_{k} P_{k}^{-} H_{k}^{\mathrm{T}}\right)}=\frac{\operatorname{tr}\left(\frac{1}{m} \sum_{j=1}^{m} d_{k-j} d_{k-j}^{\mathrm{T}}-R_{k}\right)}{\operatorname{tr}\left(H_{k} P_{k}^{-} H_{k}^{\mathrm{T}}\right)}
$$

Make the final effect approximate smooth by rooming the variance of state noise according to Equation (6).

$$
\hat{Q}_{k}=Q_{k-1} \sqrt{\beta_{k}}
$$

\subsection{Observation Interference Improvement}

The residual appears due to the difference between the filter value and observation value. The formula is as follows:

$$
e(k)=y_{k}-\tilde{y_{k}}
$$

In the formula, $y_{k}$ is the observation value and $y_{k}^{\tilde{k}}$ is the filter value.

Under the condition that the filter has no deviation, to make the mean square for the variance $e(k)$, the following equation must be satisfied:

$$
\frac{1}{\mu} \sum_{j=k-\mu+1}^{k} e(j) e(j)^{\mathrm{T}}=P_{y_{k} y_{k}}+R_{k}
$$

In the formula, $\mu$ is the size of the scroll window, $P_{y_{k} y_{k}}$ is the observation estimation variance, and $R_{k}$ is the observation noise variance matrix.

When observation interference occurs in the valuation system, the practical error will be higher than the theoretical value. The equal factors on both sides of the equal sign in Equation (9) will not exist. It is necessary to add adaptive matrix $S(k)$. The definition factor of adaptive matrix $S(k)$ is as follows: 


$$
\frac{1}{\mu} \sum_{j=k-\mu+1}^{k} e(j) e(j)^{\mathrm{T}}=P_{y_{k} y_{k}}+S(k)+R_{k}
$$

Thus, the definition of adaptive matrix $S(k)$ is as follows:

$$
S(k)=\frac{1}{\mu} \sum_{j=k-\mu+1}^{k} e(j) e(j)^{\mathrm{T}}-P_{y_{k} y_{k}}-R_{k}
$$

Because the right three variables in Equation (11) are all Gaussian variables, we can determine that the statistical characteristic is based on the $X^{2}$ distribution. We can observe whether abnormal values exist by detecting $S(k)$.

$$
\begin{cases}\operatorname{tr}(S(k))>\chi^{2}(m), & \text { abnormal value } \\ \operatorname{tr}(S(k))>\chi^{2}(m), & \text { normal value }\end{cases}
$$

In the formula, $m$ is the number of observation variables. In the case of unclear judgment standards and large data volume, the error of the above method will be larger and larger. Therefore, this paper puts forward a new judgment method to improve accuracy. By comprehensively considering the variance and residual, the trace of adaptive matrix generated by the filter can be judged adaptively each time.

The threshold value is obtained, and then the function $g(k)$ is defined to correct the state estimation.

$$
g(k)=\left\{\begin{array}{lc}
1 /\left(e(k)^{\mathrm{T}} S(k)^{-1} e(k)\right), & \text { abnormal value } \\
1, & \text { normal value }
\end{array}\right.
$$

Finally, state estimation can be modified to the following form:

$$
\hat{x}(k+1)=\hat{x}(k+1 \mid k)+K(k) g(k) e(k)
$$

Combine the improvement under state noise and observation interference. Figure 2 shows the double adaptive algorithm process.

\subsection{SOH Estimation of Improved Unscented Kalman Filtering Algorithm}

According to the equivalent circuit model, at first estimate SOC through the double adaptive UKF algorithm. Then, regard SOC as the known quantity of estimating resistance. The normal form of the state equation and observation equation of the linear discrete system is shown as follows:

$$
\left\{\begin{array}{l}
x_{k+1}=A_{k} x_{k}+B_{k} u_{k}+w_{k} \\
y_{k}=C_{k} x_{k}+v_{k}
\end{array}\right.
$$

The battery SOC can be obtained through an hour integral method.

$$
S O C_{k}=S O C_{k 0}-\frac{1}{Q_{0}} \int_{t_{0}}^{t} \eta I_{t} \mathrm{~d} t
$$

In the formula, $Q_{0}$ is the battery capacity, and then we can get the battery state space model as follows:

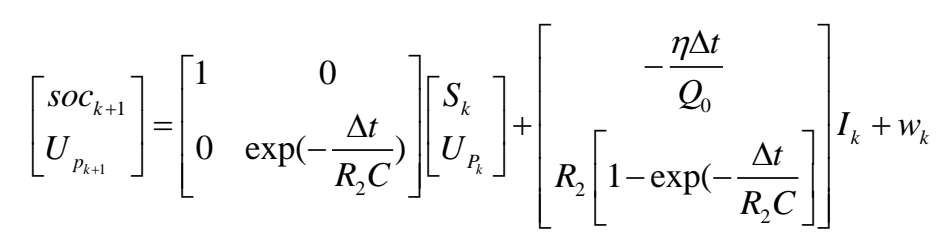




$$
U(k)=U_{O C}(k)-U_{p}(k)-R_{0} \times I_{k}+v_{k}
$$

In the formula, $w_{k}$ is the system noise, which is the error caused by system parameters' uncertainty and sampling accuracy. Its covariance is $Q$. The measurement noise is $v_{k}$, whose covariance is $R$. $U(k)$ is the observation variable $y_{k}$. The coefficient matrices of the control variable and state space model are as follows:

$$
x_{k}=\left[\begin{array}{c}
S O C_{k+1} \\
U_{P_{k+1}}
\end{array}\right], A_{k}=\left[\begin{array}{cc}
1 & 0 \\
0 & \exp \left(-\frac{\Delta t}{R_{2} C}\right)
\end{array}\right], B_{k}=\left[\begin{array}{c}
-\frac{\eta \Delta t}{Q_{0}} \\
R_{2}\left[\begin{array}{cc}
1-\exp \left(-\frac{\Delta t}{R_{2} C}\right.
\end{array}\right]
\end{array}\right], C_{k}=\frac{\partial U_{k}}{\partial X}=\left[\begin{array}{ll}
\frac{\partial U_{k}}{\partial U} & \frac{\partial U_{k}}{\partial S O C}
\end{array}\right]
$$

Combining Equations (17) and (18), we can obtain the optimal estimation of SOC through the double adaptive UKF algorithm shown in Figure 2.

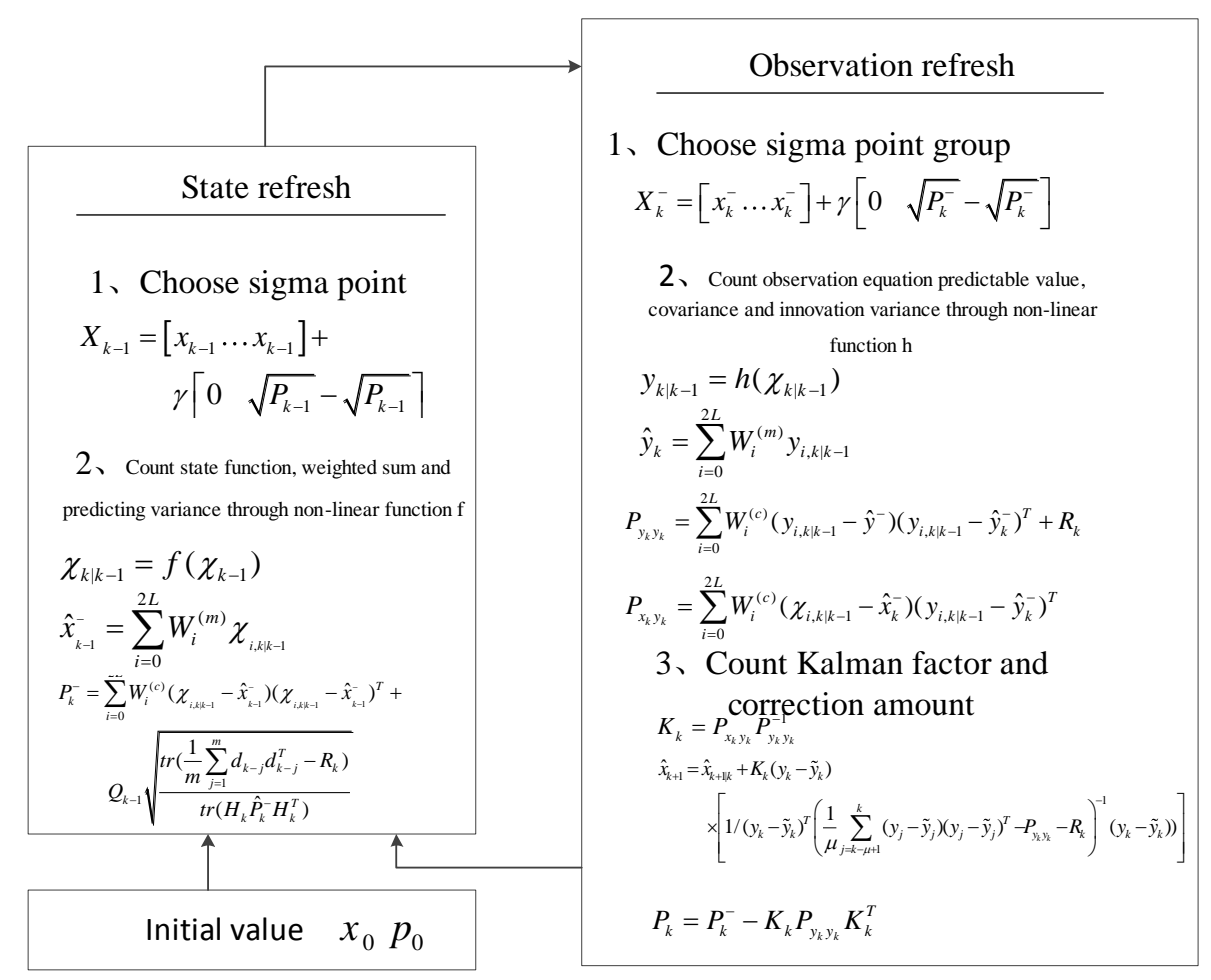

Figure 2. Double adaptive UKF algorithm flow chart

\subsection{SOH Estimation of Improved Unscented Kalman Filtering Algorithm}

The definition of battery $\mathrm{SOH}$ is as follows:

$$
S O H_{R}=\frac{R_{0}(\text { end })-R_{0}(t)}{R_{0}(\text { end })-R_{0}(0)} \times 100 \%
$$

We can determine that we need to estimate the battery's Ohm resistance while estimating $\mathrm{SOH}$. First regard the battery Ohm resistance $R_{0}$ as the system state and obtain the system state space equation as well as the output observation equation.

$$
\begin{gathered}
R_{0}^{k+1}=R_{0}^{k}+r_{k} \\
U(k)=U_{O C}(k)-U_{p}(k)-R_{0} \times I_{k}+y_{k}
\end{gathered}
$$


In the formula, the state space model was established using the ohmic internal resistance $R_{0}$ of the battery as the state variable. Among them, $U_{O C}(k)$ is the open circuit voltage of the system at the $k$ sampling moment, which is the function of $S O C$. Regard it as a known quantity. The system state variables are $x_{k}=R_{0}{ }^{k}, A_{k}=1$, and $C_{k}=\left.\frac{\partial U_{k}}{\partial R}\right|_{R=R_{k}}=I_{k}$, and $r_{k}$ is system noise, whose covariance is $Q \cdot y_{k}$ is measurement noise, whose covariance is $R$. Initialize $\hat{x}_{0}=E\left[x_{0}\right]$ and $P_{0}=E\left[x_{0}-\hat{x}_{0}\right]\left[x_{0}-\hat{x}_{0}\right]^{\mathrm{T}}$. Combining Equations (20) and (21), we can obtain the estimation method of SOH.

\section{Simulation and Experiment Results Analysis}

\subsection{Simulation Results}

To verify the double adaptive UKF algorithm accuracy for estimating SOC and SOH online, choose NP65-12 VRLA of 12V and $65 \mathrm{Ah}$ to perform the repeated charge and discharge test. The other initial settings are the following: SOC is $90 \%, U_{O C}$ is $14.09 \mathrm{~V}, R_{0}$ is $30.43 \mathrm{~m} \Omega, R_{P}$ is $8.95 \mathrm{~m} \Omega$, and $C_{P}$ is $21.2 \mathrm{~F}$. Then, estimate $S O C$ and $S O H$ through the charge and discharge test and realize the contrast of the battery actual value and estimation value before and after the algorithm improvement. The contrast of voltage, $\mathrm{SOC}$, Ohm resistance, and $\mathrm{SOH}$ estimation values and actual values before and after UKF algorithm improvement is shown in Figures 3 to 6.

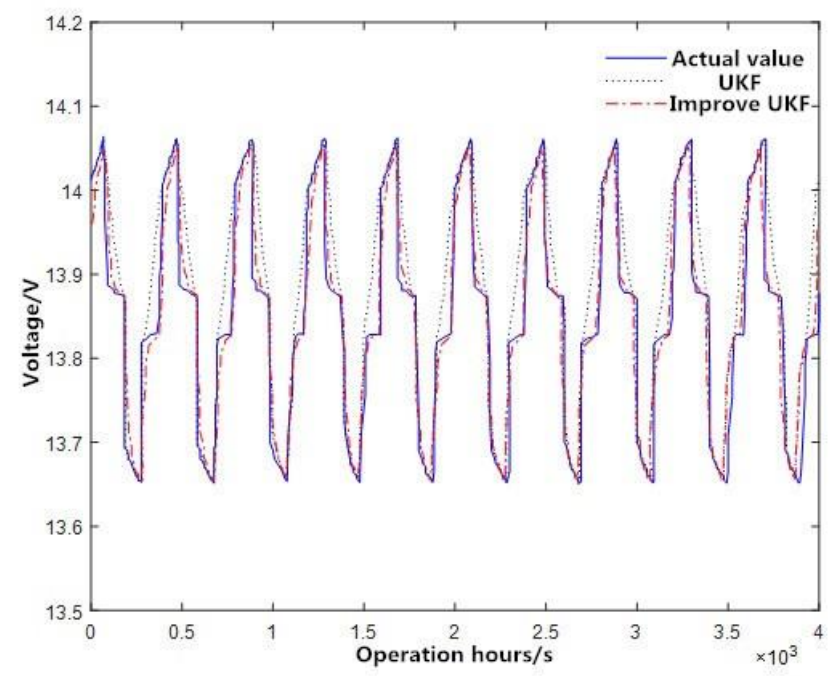

Figure 3. Contrast curve of voltage estimation and actual value before and after UKF algorithm improvement

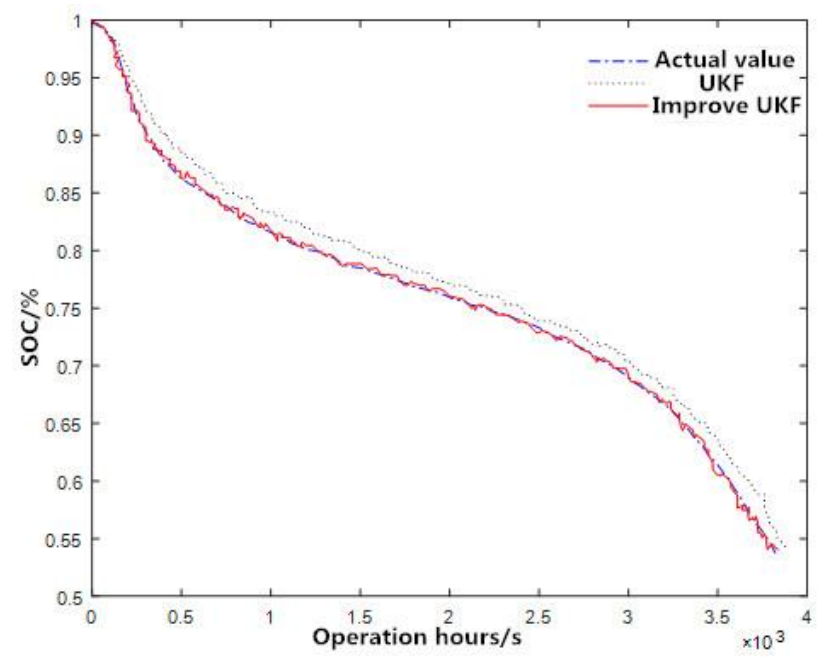

Figure 4. Contrast curve of $S O C$ estimation and actual value before and after UKF algorithm improvement 


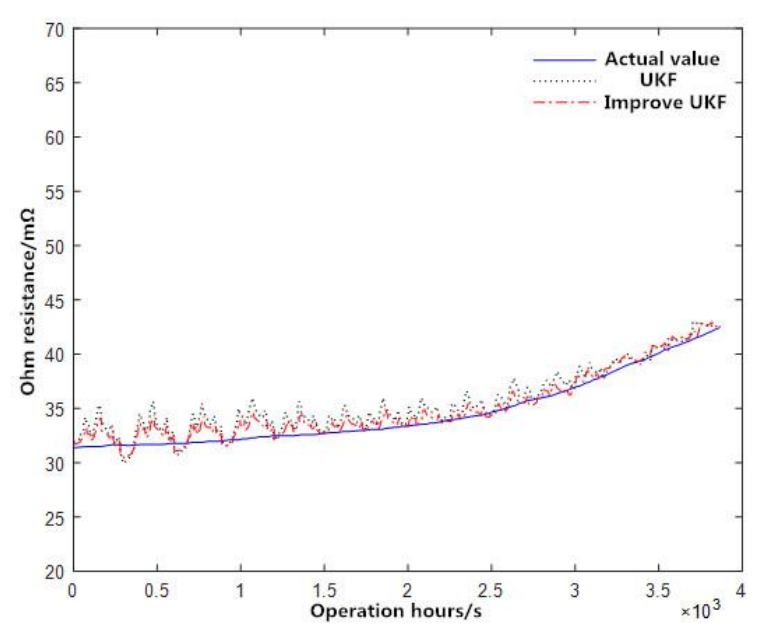

Figure 5. Contrast curve of Ohm resistance estimation and actual value before and after UKF algorithm improvement

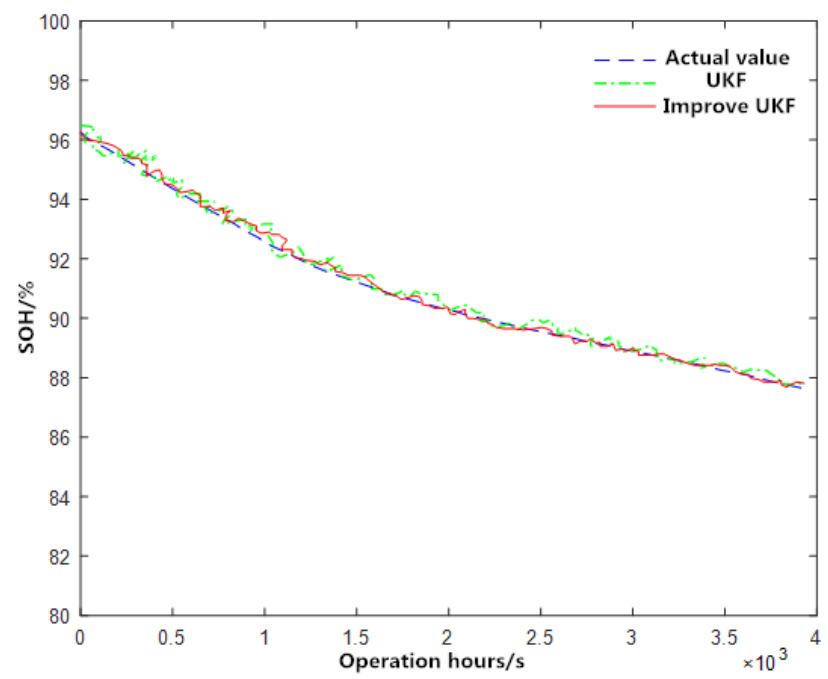

Figure 6. Contrast curve of $\mathrm{SOH}$ estimation and actual value before and after UKF algorithm improvement

We can see from Figure 4 that the average relative error of the SOC actual and estimation values in the UKF algorithm is less than $1 \%$, which is more accurate than the standard UKF. From the resistance estimation contrast curve of Figure 5, we can see that although the estimation curve fluctuation in the beginning is bigger, as time goes by, the estimation and actual value curves fit gradually in the improved UKF algorithm and the fluctuation is smaller. The fluctuation of the $S O H$ value estimated by the standard UKF algorithm is larger than the actual value. The $S O H$ value estimated by the improved UKF algorithm is closer to the actual value than the standard UKF algorithm, and the fluctuation is smaller. The $S O H$ average relative error of the improved UKF algorithm is $3.1 \%$, which is better than the estimation accuracy of the standard UKF algorithm.

\subsection{Test Results Analysis}

Regard the single battery parameters obtained online as the measurement value. To verify the reliability and accuracy of the designed test platform of estimating $\mathrm{SOH}$ online and the $\mathrm{SOH}$ algorithm of this paper, during the process of battery curve display, measure the corresponding voltage and resistance of every moment offline by using a multimeter and resistance detector. The value is regarded as the theoretical value, as shown in Table 1.

According to the theoretical and measurement values, we can draw their curves. The theoretical and measurement contrast curves of voltage, SOC, and resistance are shown in (a), (b), and (c) in Figure 7.

From the contrast curves of theoretical and measurement value, we can see that there is an error between the measurement curve and theoretical curve; however the error is very small. The biggest relative error of resistance estimation 
is around 3\%. Thus, this measurement platform satisfies the demand of measuring SOH online and verifies the accuracy of the double adaptive UKF algorithm in battery $\mathrm{SOH}$ estimation.

Table 1. Actual values of battery status parameters

\begin{tabular}{ccc}
\hline Time $(\mathrm{h})$ & Voltage $(\mathrm{V})$ & Resistance $(\mathrm{m} \Omega)$ \\
\hline 1 & 13.61 & 32.6 \\
2 & 13.82 & 33.8 \\
3 & 13.93 & 33.8 \\
4 & 14.05 & 34.1 \\
5 & 14.06 & 34.2 \\
6 & 14.09 & 34.3 \\
7 & 14.09 & 34.6 \\
8 & 14.10 & 35.0 \\
9 & 14.09 & 35.7 \\
10 & 14.10 & 35.8 \\
\hline
\end{tabular}

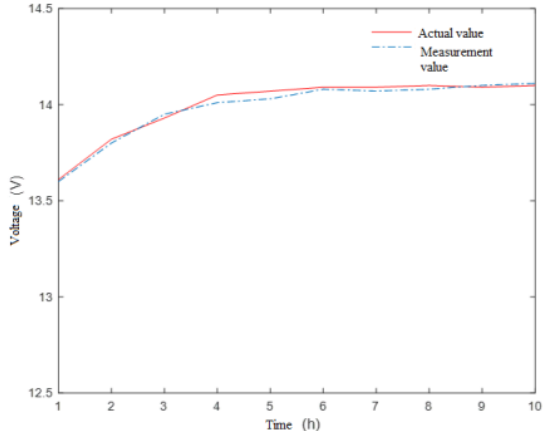

(a)

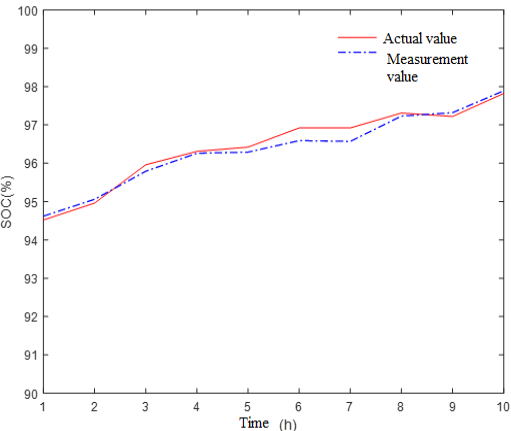

(b)

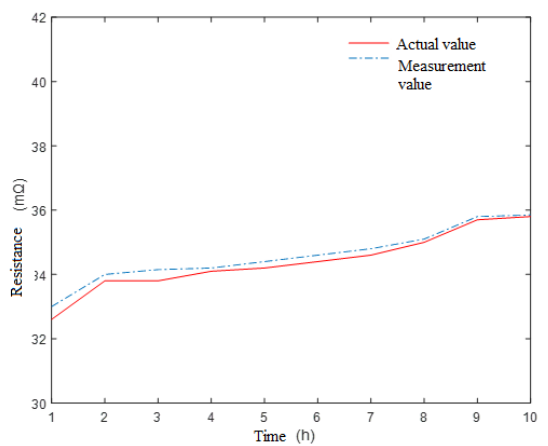

(c)

Figure 7. Contrast curves of theoretical and measurement value

\section{Conclusions}

To address the problem that battery $\mathrm{SOH}$ cannot be monitored online, this paper analyzes battery $\mathrm{SOH}$ characterization and influencing factors through experimentation. By studying the standard UKF algorithm, the UKF algorithm is optimized from the two aspects of state noise and observed interference, and then the dual adaptive UKF algorithm is obtained. Then, the state equation and output equation are built by using the Thevenin battery model. $\mathrm{SOH}$ is estimated online by using the double adaptive UKF algorithm. Simulation tests show that the SOH estimation error of this algorithm is $3.1 \%$, which meets the accuracy requirement.

\section{Acknowledgements}

This work is supported by the National Key Research and Development Program of China (No. 2016YFC0300104), Advance Research Project of Weapon Equipment (No. 41421040301), and Technological Innovation Foundation for Youth Reserve Talents of Disciplines in Science of Harbin (No. 2017RAQXJ069).

\section{References}

1. J. Liang, "Discussion on the Factors Affecting the Service Life of VRLA Battery," Guangdong Electric Power, Vol. 18, No. 2, pp. 70-76, 2005

2. C. Liang and L. Zhang, "The Present Situation of Lead Acid Battery and its Development Direction," Guangdong Chemical Industry, Vol. 33, No. 2, pp. 4-6, 2006

3. Y. Fu and T. Ding, "Online Diagnostic Technology of VRLA Battery," Contemporary Communication, Vol. 13, No. 3, pp. 5153,2006

4. X. J. Wang, "Research on Internal Resistance Monitoring Technology of VRLA Battery," Electronic Design Applications, No. 2, pp. 81-82, 2004

5. T. Z. Wu and L. P. Hu, "Research on SOC Estimation Algorithm of Power Battery based on UKF," Power Electronics Technology, Vol. 48, No. 4, pp. 23-26, 2014

6. Q. T. Wang, Y. Z. Jiang, and Y. H. Lu, "Estimation of Health Status of 18650 Lithium - Ion Battery based on UKF," Power Technology, Vol. 40, No. 3, pp. 543-546, 2016

7. L. W. Li and J. Y. Zou, "Design and Realization of Online Monitoring System for Battery," Journal of Electrotechnical Society, No. 11, pp. 7-9, 2002 
8. C. Zhu, "Online Estimation of SOC and SOH for VRLA Batteries in Standby Power System," Beijing Jiaotong University, 2010

9. X. D. Liu, "Research on Remote Monitoring System of Power Supply," Wuhan University of Technology, 2005

10. W. J. Xu, "Study on Estimation Method of Health Status of Lithium-Ion Battery for Pure Electric Vehicle," Jilin University, 2012

11. Y. F. Li, L. L. Zhou, and L. Wang, "Online Intelligent Identification and Evaluation of SOC and SOH for Electric Vehicle Storage Battery based on Operating Conditions," Measurement and Control Technology, Vol. 32, No. 4, pp. 100-104, 2013

12. J. H. Xia, Y. Y. Qin, and R. Long, "Influence of Initial Conditions of Kalman Filter on SINS Alignment," Journal of Astronautics, Vol. 31, No. 8, pp. 1933-1938, 2010

13. Z. Q. Jiang, "Research and Application of Filtering Algorithm in Autonomous Navigation," Beijing University of Posts and Telecommunications, 2014

14. Z. Liu, "Research on UKF Algorithm and Its Improved Algorithm," Central South University, 2009

15. L. Wang Lu, G. C. Li, and X. W. Qiao, "An Adaptive UKF Algorithm based on Maximum Likelihood Criterion and Maximum Expectation Algorithm," Journal of Automation, Vol. 38, No. 7, pp. 1200-1210, 2012

16. Y. Lin, X. Zhu, Z. Zheng, Z. Dou, and R. Zhou, "The Individual Identification Method of Wireless Device based on Dimensionality Reduction And Machine Learning,” Journal of Supercomputing, No. 5, pp. 1-18, 2017

17. Y. Lin, C. Wang, J. X. Wang, and Z. Dou, "A Novel Dynamic Spectrum Access Framework based on Reinforcement Learning for Cognitive Radio Sensor Networks,” Sensors, Vol. 16, No. 10, pp. 1-22, 2016

18. Y. Lin, C. Wang, C. Ma, Z. Dou, and X. Ma, "A New Combination Method for Multisensor Conflict Information," Journal of Supercomputing, Vol. 72, No. 7, pp. 2874-2890, 2016

19. Q. Wu, Y. Li, and Y. Lin, "The Application of Nonlocal Total Variation in Image Denoising for Mobile Transmission," Multimedia Tools \& Applications, Vol. 76, No. 16, pp. 1-13, 2016

20. C. Shi, Z. Dou, Y. Lin, and W. Li, "Dynamic Threshold-Setting for RF-Powered Cognitive Radio Networks in Non-Gaussian Noise,” Physical Communication, Vol. 27, No. 4, pp. 99-105, 2018 